\title{
Summary of the Public Health Network's Guidance for Tuberculosis Prevention and Control Programs in Canada
}

\author{
Lord $\mathrm{L}^{1^{*}}$ on behalf of the Public Health Network Council
}

${ }^{1}$ Public Health Agency of Canada, Ottawa, Ontario

* Corresponding author: linda.lord@phac-aspc.gc.ca

\section{Introduction}

Public health professionals engaged in the fight against tuberculosis (TB) in Canada face unique challenges due to the nature of the disease and its underlying risk factors. In 2012, provinces and territories reported 1,686 new active and re-treatment TB cases to the Public Health Agency of Canada. Foreign-born people accounted for the majority of reported TB cases, while the reported incidence rate remained highest among Canadian-born Aboriginal people (1). The purpose of this article is to summarize the key findings from Guidance for Tuberculosis Prevention and Control Programs in Canada published by the Pan-Canadian Public Health Network (2).

\section{Approach}

The Pan-Canadian Public Health Network (PHN) brings together individuals from many sectors and levels of government in Canada who are working together to strengthen public health. The work of the PHN is governed by the Public Health Network Council, which is composed of federal, provincial, and territorial government officials, including the Chief Public Health Officer of Canada and senior government officials from all jurisdictions, who are responsible for public health. The PHN ensures that Canada is better prepared for future public health events by fostering cooperative and collaborative approaches on public health matters and is accountable to the Conference of Federal/Provincial/Territorial Deputy Ministers of Health.

To identify best practices for the prevention and control of TB in Canada, an iterative approach was undertaken that included an in-depth review of TB programs in Canada. This is a summary of the full report (2).

\section{Results}

The PHN's Guidance for Tuberculosis Prevention and Control Programs in Canada describes the essential components of an evidence-based TB prevention and control program and how they can be put into practice. Part I situates TB prevention and control in a global context and outlines the goals set for Canada in conjunction with the Global Plan to Stop TB 2006-2015.

Part II describes more than 80 proven best practices for optimizing current TB prevention and control efforts through a structured TB program design based on 12 essential components (Table 1). 


\section{Table 1: Twelve essential components of TB prevention and control programs}

1. Management of cases of active TB disease

Preventing the transmission of TB requires prompt diagnosis and treatment. Best practices underscore the importance of effective case management in controlling the spread of TB, from both a prevention and treatment perspective.

2. Contact tracing and outbreak investigation

Because the contacts of infectious TB cases are at risk of progressing to active TB disease, investigations must be carried out in a timely and organized fashion. Best practices are documented step by step with special consideration given to maximizing existing public health resources.

3. Screening for latent TB infection and active TB disease

Screening should be considered for groups at high risk for active TB disease or latent TB infection. With a focus on at-risk groups, best practices are drawn from proven strategies for early preventive intervention.

\section{Surveillance and data management}

The collection, analysis, and interpretation of epidemiological data are essential features of public health practice. The Public Health Agency of Canada maintains a comprehensive surveillance system for active TB disease which is used by all orders of government to ensure continuous improvements in service delivery and the monitoring of disease trends and treatment outcomes over time.

5. TB laboratory services

The diagnosis, treatment, and prevention of TB depend on a high standard of laboratory practice. Best practices provide a blueprint for coordinating laboratory services to best support provincial and territorial TB programs.

6. Education and professional practice

Ensuring that healthcare providers have the training and knowledge they require to enable optimal TB prevention and control is an aspect of a successful TB program that is sometimes overlooked. Best practices point to a diverse range of educational opportunities supported by strong partnerships with educational institutions, training providers, and professional organizations.

7. Community-based awareness

The history of TB in Canada has had a profound impact on the beliefs, attitudes, and behaviours of Canadians most at risk for the disease. Best practices emphasize community engagement and the need to tailor awareness activities to the cultural and linguistic needs of populations at risk.

8. Monitoring and evaluation

Measuring program performance is the key to ensuring that resources are being used effectively and having the intended impact. The establishment and monitoring of performance targets has been adopted as a best practice in a growing number of jurisdictions. (Appendix IV provides examples of potential TB program objectives and performance targets based on Canadian and American experience.)

9. High-risk populations and settings

In Canada, Aboriginal peoples and the foreign-born are the two populations with the highest reported rates of TB. Other at-risk groups include the homeless and residents of long-term care facilities. Best practices focus on improving detection and management of active TB disease and latent TB infection, recognizing that the approaches for addressing TB within these groups differ in a number of respects.

10. Strategies to address emerging issues

TB-HIV co-infection and drug-resistant TB are complicating control efforts globally. Best practices focus on internationally recognized standards for care and sources of special expertise.

11. Social and other determinants of health related to TB

It has been long understood that the burden of TB is strongly related to the social determinants of health. Best practices underscore the importance of partnerships that enhance our understanding of the non-medical factors that contribute to active TB disease and latent TB infection.

12. Research

Effective TB prevention and control strategies require major investments in research and development, without which TB elimination is unlikely. 
These components are interconnected and rely on the support of highly trained personnel (Figure 1). Parts III and IV examine the partnerships and linkages in place to strengthen public health efforts in Canada and abroad.

Figure 1: Illustration of the interconnectedness among the 12 essential components of an effective TB prevention and control program

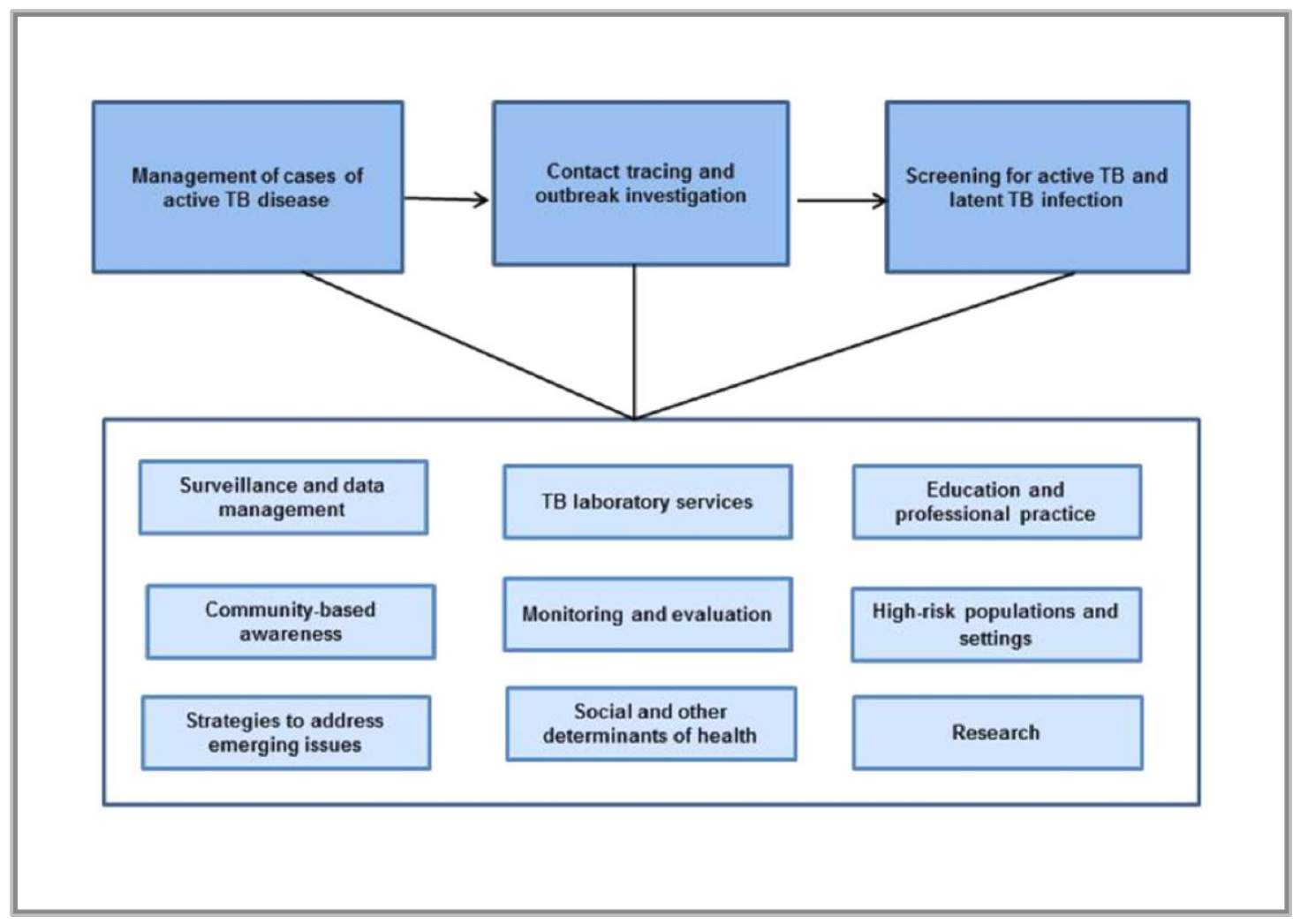

\section{Conclusion}

Addressing TB in a uniquely Canadian context requires a modern, evidence-based approach that recognizes both domestic and global disease trends. The intent of Guidance for Tuberculosis Prevention and Control Programs in Canada is to inform ongoing program delivery and development by providing decision-makers, healthcare providers, and program planners with proven best practices they can apply in their work.

\section{Acknowledgements}

The Public Health Network Council would like to thank the members of the Communicable and Infectious Disease Steering Committee, the former Canadian Tuberculosis Committee and the many individuals with the Public Health Agency of Canada and Health Canada who contributed to the development of the guidance document. 


\section{Conflict of interest statement}

There are no conflicts of interest to declare.

\section{Funding}

This work was supported by the Public Health Agency of Canada.

\section{References}

(1) Public Health Agency of Canada. Tuberculosis in Canada 2012 - Pre-release. 2013. Available at http://www.phac-aspc.gc.ca/tbpc-latb/pubs/tbcan12pre/index-eng.php

(2) Pan-Canadian Public Health Network. Guidance for Tuberculosis Prevention and Control Programs in Canada. 2012. Available at http://www.phn-rsp.ca/pubs/gtbpcp-oppctbc/index-eng.php 